\section{La planificación estratégica de la extensión universitaria. Los desafíos de construir indicadores para la intervención}

Lía Cecilia Bentolila

Área de Planeamiento de la Secretaría

de Extensión. Universidad Nacional

del Litoral, Argentina.

liabentolila@unl.edu.ar
A 100 años de la Reforma Universitaria de 1918 /

Desafíos de gestión

RECEPCIÓN: 20/06/17

ACEPTACIÓN FINAL: 24/10/17

\section{Resumen}

Se debate conceptualmente la planificación, evaluación y construcción de indicadores de proyectos de extensión de la Universidad Nacional del Litoral (UNL).

Se consideran algunos conceptos nodales: planificación estratégica, modos de intervención en el campo social y construcción de indicadores ya que estos contribuyen a generar información y contribuir a la evaluación de proyectos como siendo procesos educativos propios de lo social y a profundizar y enriquecer la extensión como dimensión sustantiva, como motor de los procesos de producción de conocimiento.

Finalmente, se presentan los indicadores construidos que contemplan la necesariedad de pensar que los proyectos impactan en el desarrollo de experiencias interdisciplinarias de intervención; el desarrollo de metodologías de identificación de problemas; la formación continua y la sociabilización de conocimientos; la producción de nuevos conocimientos y metodologías; la incorporación de contenidos relacionados con problemas sociales en el currículo y de todos los agentes universitarios a actividades de extensión, de modo de propiciar actitudes de compromiso social.

Palabras clave

- Extensión

- Evaluación

- Indicadores

- Construcción social

\section{Resumo}

É debatido o planejamento, avaliação e construção de indicadores de projetos de extensão da Universidad Nacional del Litoral conceitualmente.São considerados alguns conceitos nodais: planejamento estratégico, modos de intervenção no campo social e construção de indicadores desde que estes contribuem para gerar informação e contribuir à avaliação de projetos considerados como processos educacionais próprios do social e, afundar e enriquecer a extensão como dimensão substantiva, como motor dos processos de produção de conhecimento. Finalmente, se apresentam os indicadores construídos que contemplam a necessidade de pensar que os projetos impactam no desenvolvimento de experiências interdisciplinares de intervenção; o desenvolvimento de metodologias de identificação de problemas; a formação contínua e a socialização de conhecimento; a produção de novos conhecimentos e metodologias; a incorporação de conteúdos relacionados com problemas sociais no currículo; e a incorporação de todos os agentes universitários para atividades de extensão, propiciando atitudes de compromisso social.

Palavras-chave

- Extensão

- Avaliação

- Indicadores

- Construção social
Para citación de este artículo

Bentolila, L. C. (2017). La planificación estratégica

de la extensión universitaria. Los desafíos de

construir indicadores para la intervención. Revista

+E versión en línea, 7(7), 176-187. Santa Fe,

Argentina: Ediciones UNL. 


\section{Introducción}

Este artículo tiene como finalidad dejar esbozados ciertos indicadores que se están construyendo en la Secretaría de Extensión de la Universidad Nacional del Litoral (UNL) para la planificación y elaboración de proyectos de extensión. Pero, para ello, se transitará por algunos conceptos teóricos y políticos que se consideran necesarios. Así, en una primera instancia se comenzará por delinear el marco que se establece para definir a la extensión universitaria desde la UNL; luego se expondrán algunos conceptos teóricos sobre planificación estratégica, evaluación, modos de intervención en lo territorial y la construcción de territorialidades, y finalmente la construcción de indicadores en materia de extensión universitaria. Estos conceptos se consideran troncales al momento de enmarcar los proyectos de extensión universitaria en la genealogía de la extensión abordada desde la UNL. Los proyectos de extensión llevan más de 20 años y forman parte plena de la vida académica e institucional de la Universidad.

\section{Alcances de la extensión universitaria en la UNL}

La extensión universitaria es plenamente reconocida en el Estatuto de la Universidad, se encuentra incorporada en los Planes de Desarrollo Institucional y fortalecida por un conjunto de normativas e instrumentos de gestión que hacen posible llevar a la práctica los enfoques teóricos conceptuales y metodológicos planteados y definidos por la institución en materia de extensión universitaria. Es esta trayectoria la posibilita hoy repensar y fortalecer tanto las conceptualizaciones teóricas respecto a la extensión universitaria, así como respecto de la implementación y apropiación como política de gestión universitaria.

Definir extensión implica necesariamente incluirla como acción que se desarrolla en el territorio, construye y participa de territorialidades y por ende no puede ser concebida por fuera del campo social. Por lo tanto, la extensión universitaria tiene su "ser" en el campo social. La construcción de saberes, el diálogo de los mismos, se produce en lo social, en el territorio de intervención. Dotar al campo social de la centralidad de la extensión es imprescindible para fortalecer esta función sustantiva y al mismo tiempo enriquecer la dimensión académica, así como repensar las políticas institucionales de las tres funciones. Cabe señalar que en este artículo se resalta la función sustantiva de la extensión.

En este sentido, sostiene Gustavo Menéndez:

"la formulación de las políticas de extensión es parte de la construcción de una agenda compartida entre los actores sociales, el Estado y la comunidad universitaria con profundo sentido académico y de pertinencia social. La Universidad, mediante la extensión, promueve la apropiación social del conocimiento y la democratización del capital social y cultural, aspectos claves para contribuir a la transformación social, integrándose de este modo al medio social del cual se nutre y al que permanentemente contribuye, consciente y comprometida con las problemáticas sociales, culturales y productivas de la región en la que se encuentra inserta". (2013:84)

La extensión denota la relación interactiva que la Universidad plantea con su medio en las dimensiones sociales, culturales y productivas. Considerada en su misión sustantiva, no solo se ha conceptualizado sino que, desde la Secretaría de Extensión se han definido sus dimensiones de análisis y una determinada organización de gestión para la planificación de sus acciones. Las dimensiones intrínsecas y constituyentes de la extensión se refieren a:

- Académico-institucional, como es reconocer la función académica que aporta la extensión a la construcción y la apropiación social del conocimiento y a generar dispositivos de gestión que apuesten al fortalecimiento de las instituciones democráticas y de las políticas públicas. Formar actores universitarios con compromiso social y contar con un presupuesto adecuado a las necesidades de desarrollo.

- Pedagógica, que implica los cambios que se producen en los procesos de enseñanza y aprendizaje cuando se ponen en juego teorías, perspectivas y metodologías en situaciones "reales". Incluye al conocimiento académico cuando es pertinente y relevante para situaciones o actores determinados (apropiación social).

- Social, es decir, la Universidad como institución social que construye y se construye en las dinámicas sociales, culturales y productivas, en un tiempo y territorio establecidos. Las prácticas de extensión se definen cuando tienen implicancia social.

- Comunicacional, dado que se inscribe en un campo de relaciones entre sujetos (académicos, sociales, productivos) que ponen en juego significaciones, percepciones, poderes e intereses que es necesario reconocer y definir.

- Política, puesto que toda acción de extensión tiene por objetivo transformar una situación determinada. Esto supone interpelar al Estado y a las políticas públicas y construir agendas de trabajo sobre aspectos relevantes de la "cosa pública".

A partir de estas dimensiones se han definido también los ejes estratégicos en que se basa la gestión de la Secretaría en el marco del Plan de Desarrollo Institucional de la UNL. Se trata de: - Institucionalización y reconocimiento académico de la extensión, eje en el cual se focalizan las prácticas de educación experiencial en extensión y se busca el reconocimiento académico de las mismas. - Integración de la extensión con la docencia y la investigación, en tanto a través de este eje se busca consolidar las funciones sustantivas a través de la conversión de proyectos de extensión e investigación.

- Construcción de la agenda territorial con distintos actores, ya que consensuar agendas con los actores sociales es de suma importancia a los fines de definir y delimitar los requerimientos en ese aspecto. 


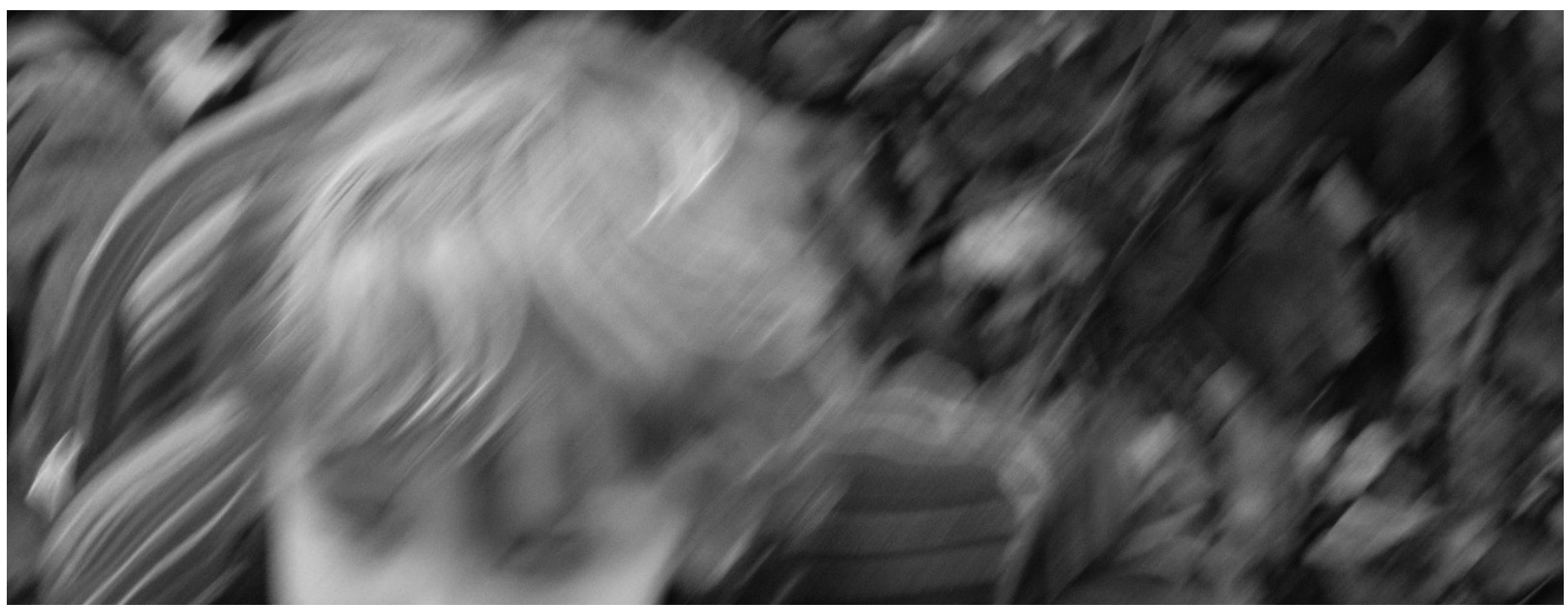

(c) Cecilia lucci

- Análisis y aportes a las políticas públicas, pues es una clara política de extensión su imbricamiento con las políticas públicas. Toda acción de extensión implica un anclaje en alguna política pública, sea para su fortalecimiento o bien para denotar la necesidad de construcción si estuviera ausente.

- Consolidación del Sistema Integrado de Programas y Proyectos de Extensión, sistema que estructura, organiza y coordina las iniciativas de extensión con intervención en el medio social a través de proyectos y programas.

- Fortalecimiento de la política editorial, la cual se centra en una estrategia de socialización del conocimiento.

- Formación y capacitación en extensión, eje que se sostiene a partir de instancias de capacitación en las que pueden encontrarse desde propuestas de formación profesional hasta talleres de capacitación para el trabajo.

- Internacionalización de la extensión, dado que las políticas de internacionalización de la extensión promueven acciones de cooperación y de movilidad. Se participa activamente de las redes regionales e internacionales de extensión.

- Consolidación de líneas estratégicas de comunicación.

- Planeamiento y evaluación de la extensión, lo cual permite valorar el funcionamiento de las actividades de extensión y obtener información relevante que se constituya en insumo para la planificación.

Habiendo definido en líneas generales las dimensiones y ejes estratégicos de extensión se enunciará específicamente el área de gestión encargada del planeamiento, sistematización, seguimiento y evaluación de extensión.

\section{Área Planeamiento y evaluación de la extensión}

En el año 2014 se incorporó como área de gestión de la Secretaría de Extensión la de Planeamiento y evaluación de la extensión, cuya misión es, como fundamental, la articulación con la Secretaría de Planeamiento de la UNL con el propósito de llevar adelante los objetivos propuestos, en materia de extensión, en el marco del Plan de Desarrollo Institucional. Este último posee tres Líneas de Orientación Principal (LOP) presentadas como grandes políticas guías de la gestión institucional, encuadradas en el compromiso social de expansión del saber, del conocimiento y de la cultura en diálogo permanente con la sociedad. Así, se contempla un abordaje sistémico de la estructura de la Universidad, la diversificación de sus funciones, la ampliación de áreas de conocimiento y compromiso con el medio social.

En lo que se refiere a la extensión universitaria, el eje de mayor injerencia se corresponde con la LOP II, respecto de la alta calidad en enseñanza, investigación y extensión del conocimiento. Dentro de esta línea se plantean cuatro objetivos generales:

“1) Afianzar la democratización de la educación superior, recreando en forma permanente dispositivos de acceso y permanencia igualadores de oportunidades. Desarrollar, en un marco de integración y transversalidad, un arco diverso de propuestas curriculares flexibles y de alta calidad en las distintas disciplinas, niveles y modalidades, a nivel nacional e internacional, que les permita a sus graduados un eficaz desempeño ya sea profesional o científico.

2) Fortalecer el desarrollo de actividades de investigación y de extensión, proyectándose al medio social y productivo como factor estratégico del crecimiento institucional, 
articulando acciones con organismos regionales, nacionales e internacionales y potenciando la producción de conocimientos en todas las ramas del saber.

3) Establecer estrategias de formación, perfeccionamiento y organización del personal académico que garantice la cobertura eficaz y versátil de las misiones y funciones institucionales de docencia, investigación y extensión y sus articulaciones en y entre todas las Unidades Académicas, Escuelas e Institutos.

4) Generar capacidades institucionales y técnicas para gestionar el arco de propuestas curriculares de manera que el desempeño de los estudiantes convalide las previsiones de los planes de estudios tanto en los alcances de la formación teórica y práctica cuanto en su duración y efectividad". (UNL, 2010, s/n)

Así es que el área articula sus políticas de gestión con la Secretaría de Planeamiento en función de asistir a las diferentes áreas de la Secretaría de Extensión en la ejecución del PDI llevado adelante por la UNL. Asimismo, identifica perfiles de proyectos y en el diseño, desarrollo y evaluación de los proyectos y acciones que involucran a esta Secretaría. Contempla el Programa de autoevaluación y evaluación externa de la extensión universitaria y coordina el Plan de mejoras que surja de dicho proceso. Lleva adelante el sistema de información actualizada y sistematizada de la Secretaría de Extensión y participa de estudios exploratorios en el territorio en el que se interviene con aportes de Observatorio Social. De esta manera, a partir de la generación del área de Planeamiento y evaluación se profundizó la consolidación del monitoreo y evaluación como herramientas que favorecen el seguimiento de, diremos ahora, acciones de extensión en general, para luego focalizar específicamente en los modos en que se planifican, sistematizan y evalúan los proyectos de extensión.

Se sostiene que es de vital importancia realizar una planificación, lo más ajustada posible, de las acciones que se emprenden. Los porqués pueden ser muchos y diversos, pero si hay algo que define a la extensión universitaria de la UNL es que su misión es generar acciones en conjunto con los actores/agentes sociales. Y esta generación es parte de un proceso social que antecede y trasciende a la participación de las acciones específicas. Por lo tanto, la planificación de las acciones debería contemplar el hecho de que ello deviene de un contexto social particular. Y, de la mano de la planificación, está la evaluación, que no es un fin en sí, y tampoco podemos sostener que sea el medio único e idóneo para superar los problemas que requiere el medio social. Pero, sin duda,

"ella contribuye a aumentar la racionalidad en la toma de decisiones, identificando problemas, seleccionando alternativas de solución, previendo —-dentro de lo posiblesus consecuencias, y optimizando la utilización de los recursos disponibles" (Cohen 1992:282).
Consecuentemente, podemos decir que los procesos de monitoreo y evaluación no representan solo una herramienta de gestión sino que constituyen procesos de aprendizaje a partir de una información obtenida. Por otra parte, la construcción de indicadores es una instancia fundamental de los procesos de evaluación, en tanto facilita la búsqueda de respuesta a los interrogantes planteados y a los objetivos que sustentan la evaluación (Xinia Picado, 1997).

A continuación, se presentarán los lineamientos teóricos que, desde la gestión de la Secretaría de Extensión, se consideran los más adecuados para el monitoreo, sistematización, planificación y evaluación de proyectos para, de allí, comenzar a plantear posibles indicadores para los mismos y modos de abordaje e interpretación de las intervenciones en el campo social.

\section{Referencias teóricas para la planificación, evaluación y monitoreo de proyectos de extensión. Planificación estratégica como método para planificar proyectos}

No es la intención de este artículo disertar sobre el soporte teórico para planificar, evaluar y monitorear proyectos de extensión en la UNL, pero sí dejar enunciadas ciertas perspectivas teóricas que desde la Secretaría de Extensión de la UNL se consideran importantes.

En materia de planificación de acciones involucradas en un proyecto de extensión, se piensa que el método de la planificación estratégica situacional planteada por Carlos Matus es valedero en esta materia puesto que permite abordar la tarea de explicación y análisis situacional integrando, en tanto que es primordial primero comprender el contexto situación en el que se enmarca un proyecto para a partir de allí planificar las tareas. También este método aborda desde un inicio la dimensión exploratoria, descriptiva y explicativa y evita el riesgo de entenderlas como etapas secuenciales, o de identificar la tarea con alguna de ellas. Todo proyecto de extensión comprende alguna temática que es considerada como problemática para los actores sociales involucrados, por ende, se debe explorar, describir y explicar esa temática considerando los puntos de vista de los actores sociales que contempla, quienes piensan que esa temática es un problema para ellos, para la comunidad, barrio, institución, etc. Entender el contexto situacional implica, por lo tanto, comprender las diferentes racionalidades, conocimientos y perspectivas de la realidad. Para comprender el contexto y hacer circular las diferentes racionalidades se debe construir un lenguaje común en un espacio caracterizado por la diversidad, que admita la formulación de estrategias de intervención estrechamente ligada al análisis situacional, evitando el riesgo frecuente de trabajarlos como compartimentos estancos. 
Este artículo no se propone detallar minuciosamente el método de la planificación estratégica pero sí referenciarlo para contextualizar desde dónde son pensados, a nivel de gestión, los proyectos de extensión.

\section{Evaluación como proceso de aprendizaje y monitoreo como acompañamiento}

Desde la visión teórica del planeamiento y la evaluación, respecto de las políticas de gestión de extensión de la UNL se otorga relevancia al proceso de evaluación desde dos sentidos diferentes: por un lado, como seguimiento y control y, por otro, como profundización del conocimiento disponible. Este último punto es clave en el desarrollo de la planificación de nuevas acciones y para delinear los ejes de trabajo futuro.

En este apartado se resalta la importancia que tiene la evaluación de proyectos de extensión entendida como un proceso. Cohen lo expresa como un "proceso organizativo para mejorarlas actividades todavía en marcha y ayudar a la administración en la planificación, programación y toma de decisiones futuras" (1992:77). El énfasis en el carácter procesal de la evaluación de proyectos de extensión destaca dos cuestiones: que no se trata de un hecho ajeno y separado del proyecto en cuestión, y que el desarrollo de un proyecto se encuentra inmerso en un proceso social. Este abordaje permite mostrar la trayectoria de la planificación estratégica en la que se basan las políticas de extensión universitaria de la UNL. La evaluación permanente de los proyectos de extensión, ejecutadas y en ejecución permite realizar un análisis, en el sentido de generar una evaluación, y una planificación, tanto de los procesos como de los resultados, con el fin de valorarlos y, a partir de ello, fortalecer las acciones y políticas de extensión que se llevan a cabo desde la universidad.

En tal sentido, se toman como soporte teórico para abordar conceptualmente a la evaluación los aportes de Nirenberg, quien la conceptualiza como "una actividad programada de reflexión sobre la acción, cuya realización puede proponerse para antes, durante o después de la implementación de una política, programa o proyecto" (2005:32).

Desde la gestión de la Secretaría de Extensión se piensa que es sumamente importante evaluar los proyectos desde el aporte de sus intervenciones, así como las repercusiones de estas en el tiempo, no solo mientras dure el proyecto sino luego. Esto es, las consecuencias favorables $y / o$ no favorables de esas intervenciones sin ya la participación de los actores académicos.

Para evaluar se deben llevar a cabo procedimientos sistemáticos de recolección, análisis e interpretación de información a través de comparaciones respecto de parámetros definidos, ya que su finalidad es "emitir juicios valorativos fundamentados y comunicables, sobre las actividades y los resultados (...) de las intervenciones sociales y formular recomendaciones que permitan decisiones orientadas a ajustar la acción." (Nirenberg, 2009:32).

Cabe aclarar que la evaluación debe estar enfocada en diferentes etapas del proceso: o bien preceder a la acción (ex ante), en simultáneo con el desarrollo dela acción (concurrente), o una vez finalizado, con eje en los efectos del mismo (ex post).

De manera concomitante con la evaluación se da el monitoreo, y aquí se resaltan los aportes teóricos de Di Virgilio (2012), en tanto este representa el seguimiento para la revisión periódica de los avances de proyectos. Constituyen una herramienta de gestión, así como un "un insumo indispensable para la gestión administrativa y estratégica” (2012:40).

El monitoreo implica un trabajo continuo para reencaminar el accionar hacia la concreción de los objetivos establecidos, mientras que la evaluación "permite la formulación de conclusiones acerca de lo que se observa a una escala mayor, aspectos tales como el diseño del proyecto y sus impactos, tanto aquellos previstos como no previstos" (45).

Si se han escogido las definiciones de planificación, evaluación y monitoreo anteriores es porque se considera que el abordaje de acciones de extensión universitaria en general y de proyectos de extensión en particular no puede pensarse de manera fragmentaria ni desvinculada tanto de los lineamientos políticos institucionales de una universidad como de los requerimientos sociales existentes en una sociedad.

Ya destacados los conceptos de planificación, evaluación y monitoreo en lo que respecta acciones y proyectos de extensión $y$, habiéndolos relacionado, enmarcados en procesos sociales, a continuación nos referiremos a la conceptualización de lo que se entiende por espacio social, concepto importante a tener en cuenta ya que se planteó que para planificar y evaluar se debe contemplar el contexto situacional en donde se desarrolla un proyecto de extensión.

\section{Espacio social}

Desde la UNL, cuando se planifican acciones de extensión universitaria en general y proyectos en particular, se deben atender a las dimensiones de espacialidad-temporalidad, esto significa considerar la complejidad del espacio. Para pensar estas categorías desde el punto de vista social se toma de referencia a M. Augé (2000) y Pierre Bourdieu (1984) puesto que el Área de Planeamiento y evaluación de la Secretaría de Extensión de la UNL considera que se debe tratar al espacio como un lugar antropológico en donde se interviene. Por lo tanto, el espacio es 
la evaluación debe estar enfocada en diferentes etapas del proceso: o bien preceder a la acción (ex ante), en simultáneo con el desarrollo dela acción (concurrente), o una vez finalizado, con eje en los efectos del mismo (ex post)

la construcción de los lugares y estos no son ni más ni menos que la conformación de prácticas colectivas e individuales (lo que más adelante se llamará de construcción de territorialidades). Así es que, al momento de planificar acciones de extensión universitaria se atenderá a la singularidad de lo colectivo, las individualidades y la apuesta a la construcción de una generalidad a partir de las particularidades existentes.

Considerando al espacio como lugar antropológico, las

intervenciones en materia de extensión universitaria implican que en ese espacio se producirá un intercambio de saberes y, si fuera el caso, una apropiación social de los conocimientos. De allí que se tomará como referencia la conceptualización bourdiana de espacio social.

El espacio social es, en primer lugar, un espacio multidimensional en el cual se dan luchas simbólicas por parte de los agentes y en donde se pone en juego nada menos que la representación del mundo social, el cual se construye sobre la base de principios de diferenciación o de distribución.

"constituidas por el conjunto de las propiedades activas dentro del universo social considerado, es decir, capaces de conferir a su detentador la fuerza, el poder en ese universo el poder de ese universo." (Bourdieu, 1984:28)

Para Bourdieu, el espacio social es un sistema de posiciones sociales que se definen las unas en relación con las otras. Así, el valor de una posición se mide por la distancia social que la separa de otras posiciones sociales, ya sean inferiores o superiores. Es decir, el espacio social es un sistema de diferencias sociales jerarquizadas en función de un sistema de legitimidades socialmente establecidas y reconocidas en un momento determinado. No obstante, por ser el campo social multidimensional, como ya se dijo, se presenta como un conjunto de campos relativamente autónomos, aunque articulados entre sí. Un campo, por lo tanto, es una esfera de la vida social que se ha ido autonomizando progresivamente a través de la historia en torno a cierto tipo de relaciones sociales, de intereses y de recursos propios, diferentes de los de otros campos. En tal sentido, la estructura de cada campo social está conformada por las relaciones entre los sujetos que son parte de dicho campo, por lo que no son estáticos sino dinámicos y se encuentran en constante producción. De allí que el campo se define como una red o una configuración de relaciones objetivas entre posiciones diferenciadas, socialmente definidas y en gran medida independientes de la existencia física de los agentes que las ocupan. La especificidad de cada campo está determinada, según Bourdieu, por el tipo de capital que posee cada sujeto.

Un campo se encuentra designado por la existencia de un capital común y la lucha por su apropiación. Entendido como una arena dentro de la cual hay un conflicto entre los actores por el acceso a los recursos específicos que lo definen, el campo posee una estructura dada por las relaciones que guardan entre sí los actores. De manera tal que un campo social consiste en un sistema estructurado de posiciones sociales, a la vez que un sistema estructurado de relaciones de fuerzas entre estas posiciones. Lo anteriormente expuesto sirve como base teórica para reflexionar en cuanto a cómo se pueden abordar los procesos sociales, las maneras de mirar-ver las realidades. En tal sentido, la teoría sociológica de Bourdieu sirve a los fines de pensar la extensión universitaria en la medida en que ninguna acción de extensión se puede planificar (por lo menos así se la piensa desde las políticas universitarias de la UNL) si no se reconoce a los otros con los que se coproduce saberes, con los que se los intercambia.

Por lo tanto, para planificar acciones tendientes a la producción y/o intercambio de conocimientos se debe tener en cuenta el 
espacio, es decir el lugar donde se conjugan "identidad y relación" (Augé, 2000), lo que conlleva a identificar los tipos de capitales que se entretejen allí.

En síntesis, tomar al espacio no como un lugar meramente físico sino además como un lugar antropológico conlleva a la idea de territorialidades, desterritorializaciones, reterritorializaciones (Deleuze, Guattari, 1994).

A continuación, se retrabajarán los conceptos de territorio y territorialidades planteados desde el punto de vista filosófico por Deleuze y Guattari y se los decontruirá (parafraseando a estos autores) para aproximarlos a la extensión universitaria.

\section{Conceptualización de territorio-territorialidades}

Se ha acordado en definir al territorio como un espacio socialmente construido, lo que conlleva a no limitarlo al lugar físico. En él no solamente se desarrollan las intervenciones de extensión sino que incluye a los actores y agentes que lo componen. La complejidad de interacciones que rodean y definen al territorio determinan que se encuentra en permanente transformación. Se resalta la interacción e intercambio de saberes que existen en el territorio como condición necesaria para el conocimiento de este, así como para el accionar transformador en el mismo.

En tal sentido, el territorio es el ámbito en donde se producen y/o se reproducen prácticas y representaciones sociales a través de las cuales los sujetos intervienen a nivel material y simbólico desde el lugar que ocupan; al no ser algo fijo el territorio implica la necesidad de entender los escenarios que se ponen permanentemente en juego. Es por eso que se comprende al territorio desde la perspectiva simbólica y desde un enfoque relacional y complejo, lo cual lo hace ser un punto central a conocer a la hora de definir acciones de intervención, espacios y agentes privilegiados para ellas. A partir de este posicionamiento teórico se puede pensar, en sentido operativo, de qué manera se construyen estrategias para llevar a cabo intervenciones sociales - acciones de extensión- en espacios microsociales.

Se puede pensar que algunas estrategias en/para extensión universitaria deben implicar necesariamente articulaciones intra e interinstitucional en donde se consideren los objetivos, las necesidades o, mejor dicho, los requerimientos sociales que se producen en el espacio social así como tener en consideración la manera en se establecen y se fortalecen los lazos personales. Por lo tanto, al momento de pensar o abordar territorialidades se debe considerar el aporte de las mismas al desarrollo microsocial, en donde la generación de procesos colectivos propicie el fortalecimiento de lazos, el respeto a la diversidad, el diálogo y la solidaridad como así también contribuyan a la construcción de políticas públicas desde la voz de los propios agentes involucrados. Para poder pensar "intervenciones en territorio" desde la extensión universitaria se consideran necesarias y preliminares algunas concepciones, a saber: toda relación social tiene ocurrencia en el territorio y se expresa como territorialidad. El territorio es el escenario de las relaciones sociales y no solamente el marco espacial que delimita el dominio soberano de un Estado. El territorio es el espacio en donde se manifiestan y se construyen relaciones de poder. a) En este sentido deben ser considerados los distintos tipos de "relaciones sociales" que se dan en ese escenario que las propician. El territorio es, según Carballeda, ese lugar "donde se despliega el guión de la trama social, donde sujeto y su entorno prueban la vulnerabilidad o eficiencia de sus lazos sociales" (2005:2).

b) El territorio es el espacio donde tienen lugar procesos socioeconómicos como sistema de relaciones constituido por grupos interdependientes, atravesados por relaciones de poder y procesos socioculturales, en términos de identidad colectiva, que hacen al sentido de pertenencia. Los procesos socioeconómicos y los sistemas de relacionamiento se sustentan desde el punto de vista teórico con lo desarrollado anteriormente respecto de la teoría sociológica de Bourdieu en lo concerniente a posiciones sociales, relaciones de poder y la circulación de capitales dentro del entramado social.

c) El territorio es una construcción social y su conocimiento implica el conocimiento y comprensión del proceso de su producción. Este conocimiento, a su vez, requiere poner en diálogo los saberes provenientes del mundo académico, como los que manejan los agentes sociales involucrados en el territorio y que se reflejan tanto en las prácticas que despliegan como en sus representaciones sociales.

d) El territorio es el espacio en donde concurren y se sobreponen distintas territorialidades locales, regionales, nacionales y mundiales, con intereses distintos, con percepciones, valoraciones y actitudes territoriales diferentes, que generan relaciones de complementación, de cooperación y de conflicto. Comprender el territorio en donde se realizarán producciones académicas — de extensión- es respetar y conocer las diferencias que constituyen y dan identidad a un territorio.

e) El territorio no es fijo sino móvil, mutable y desequilibrado. La realidad geosocial es cambiante y requiere permanentemente nuevas formas de organización territorial. El sentido de pertenencia e identidad, el de conciencia regional, al igual que el ejercicio de la ciudadanía y de acción ciudadana, solo adquieren existencia real a partir de su expresión de territorialidad.

f) El territorio constituye "un espacio de inscripción". Las intervenciones en/de extensión se construyen considerando los territorios tatuados por las huellas de la historia, de la cultura y del trabajo humano. No se puede desconocer que para producir un proceso de intervención social se debe considerar que esta implica intercambios de saberes, de escenarios y de territorios. Estos intercambios derivan en formas diversas de producción de subjetividades. Como lo expresa Carballeda: 
"La subjetividad (...) se construye y de-construye en un movimiento que se expresa en el propio devenir de la cultura, de la cotidianeidad, de una compleja trama móvil de significaciones, signada, en este caso por la noción de problema social, que en definitiva convoca a la intervención". (2005:7)

Considerar la "transformación" de/en los espacios sociales implica atender a la producción de subjetividad, en tanto esta se construye en la intersubjetividad al interior de una cultura. Dicho de otra manera, la singularidad da cuenta de simbolizaciones que son histórico-sociales. De allí que los vínculos entre la universidad y los agentes sociales no sean naturales ni continuos sino que, por el contrario, resultan profundamente complejos.

Una vez definidos conceptualmente los espacios sociales, los territorios, las territorialidades y las intervenciones, o sea, habiendo identificado la complejidad de la cual formamos parte, puesto que esta nos constituye subjetivamente, se trabajará en la siguiente sección la construcción de indicadores para la evaluación de proyectos de e extensión entendidos como proyectos que implican y están implicados en procesos sociales.

\section{Construcción de indicadores}

Apostar a la construcción de indicadores para medir resultados de acciones de extensión y, más aún, los procesos por los que atraviesa un proyecto de extensión no es una tarea fácil y se deben definir adecuadamente las variables que, por ser sociales, no son inmutables y pueden no perdurar en el tiempo.

En este apartado se presentarán algunas consideraciones teóricas en cuanto al concepto de indicadores y se arrojarán los primeros resultados elaborados por el Área de Planeamiento y Evaluación con referencia a la construcción de indicadores para la evaluación de proyectos de extensión.

A nivel teórico, la definición de indicadores es de suma importancia en tanto y en cuanto en estos radica la necesidad y el interés de relevar un fenómeno (o varios) a partir de elementos observables empíricamente.

Como se expresó anteriormente, los indicadores representan la base sobre la que se construye el sistema de evaluación y monitoreo. Es por esto que se parte de la definición de indicadores como "medidas directas o indirectas de un evento, condición, situación o concepto, y permiten inferir conclusiones apreciativas acerca de su comportamiento o variación" (Di Virgilio, 2012:47).

Pensar en proyectos de extensión universitaria implica necesariamente pensar que estos deben impactar en el desarrollo de experiencias interdisciplinarias de intervención, en las metodologías de identificación de problemas y requerimientos sociales, en la formación continua y la sociabilización de conocimientos, en la producción de nuevos conocimientos y metodologías; en la incorporación de contenidos relacionados con problemas sociales en el currículo y de todos los agentes universitarios (estudiantes, graduados, docentes y no docentes) en actividades de extensión, propiciando actitudes de compromiso social.

Precisamente, en función de la centralidad de los proyectos en el desarrollo de la extensión universitaria, resulta fundamental impulsar el monitoreo y la evaluación de las acciones por estos ejecutadas. Como se mencionara en la sección referida a la evaluación y monitoreo, la primera puede realizarse en diferentes etapas (ex ante, concurrente y ex post). La perspectiva aquí expuesta se enmarca en la evaluación ex-post. Hart (2011) sostiene:

"La evaluación ex-post, que estudia el proceso y el producto del proyecto o solo el producto, puede ser realizada también tanto por los responsables de la puesta en práctica del proyecto como por las autoridades que deben dar su aprobación y decidir, si correspondiere, sobre la iniciación, la continuidad, la interrupción o la finalización del proyecto. Los dos niveles de evaluación, el primero, institucional y el segundo, estratégico, en virtud de que persiguen diferentes objetivos, requieren la definición de indicadores específicos para ese nivel". (2011:34-58) 


\section{Algunos datos y antecedentes para la construcción de indicadores}

Como es sabido, la UNL viene ejecutando proyectos de extensión desde hace 20 años. En todo este tiempo se ha venido trabajando y retrabajando las herramientas metodológicas mediante las cuales son elaborados los distintos tipos de proyectos de extensión. A continuación, se muestra en la Figura 1 la sistematización de 482 proyectos de todo tipo que se realizaron en el período 2008-2015. En la actualidad se han modificado los tipos de proyectos que pueden presentarse. Ahora cabe la posibilidad de elaborar cuatros tipos de proyectos de extensión con diversos alcances y objetivos. Uno es el PEIS, Proyecto de Extensión de Interés Social (aquel que se refiere principalmente a abordar una temática/problemática que sea primordialmente de interés social y en el cual es el actor social quien requiere que se tome el tema). Otros son las AET, Proyectos de Acción al Territorio (que se basa en la generación de multiplicadores de actores sociales para el abordaje de una temática), y las PEEE, Proyecto de Extensión de Educación Experiencial (que es una propuesta curricular de las carreras de grado que favorece la creación de espacios de aprendizaje, en la práctica, de saberes y actitudes para abordar profesionalmente diferentes situaciones sociales). Por último, el PEII, Proyecto de Extensión de Interés Institucional (que busca generar capacidades en el medio social para garantizar la sustentabilidad en el tiempo, una vez que el proyecto haya concluido).

En la Figura 2 se puede apreciar la agrupación de proyectos en el período 2008-2015 con presencia de la nueva modalidad de proyectos (PEEE). Este tipo de proyectos reemplaza al PEC. A continuación se relatará brevemente el recorrido para la elaboración de indicadores de extensión que llevó a cabo el Área de Planeamiento y Evaluación de la Secretaría de Extensión de la UNL. En primer lugar, se definieron tres variables para considerar la generación de indicadores para evaluación de proyectos de extensión en el período 2008-2015: tipo de proyecto; alcances y objetivos respectivos; articulaciones con actores sociales (instituciones). Se registraron unas 1021 articulaciones con instituciones (contando con una amplia información: ubicación territorial, tipo de organización, etc.) con las que los diversos proyectos de extensión se vinculan; los tipos de proyectos según sus modalidades y el anclaje territorial entre otras informaciones. Pero, al momento de querer monitorear el proceso del proyecto en sí, sus logros parciales, sus resultados finales, nos encontramos con que no estábamos contando con un instrumento metodológico que nos permitiera tal análisis, por lo que decidimos reformular los formatos de presentación de los proyectos y crear variables coherentes para monitorear el proceso de ejecución de los proyectos. De allí que se definan tres dimensiones que deben

Figura 2. Proyectos según modalidad, años 2008-2015

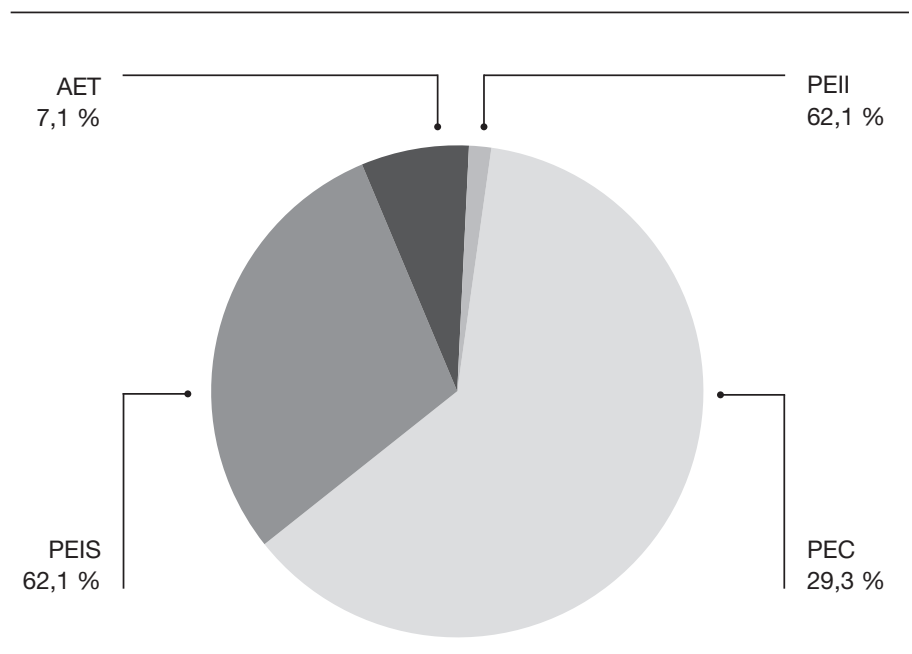

Fuente: Área de Planeamiento y Evaluación de la Secretaría de Extensión, UNL.

Figura 1. Distribución de proyectos de extensión por año según modalidad. Período 2008-2015

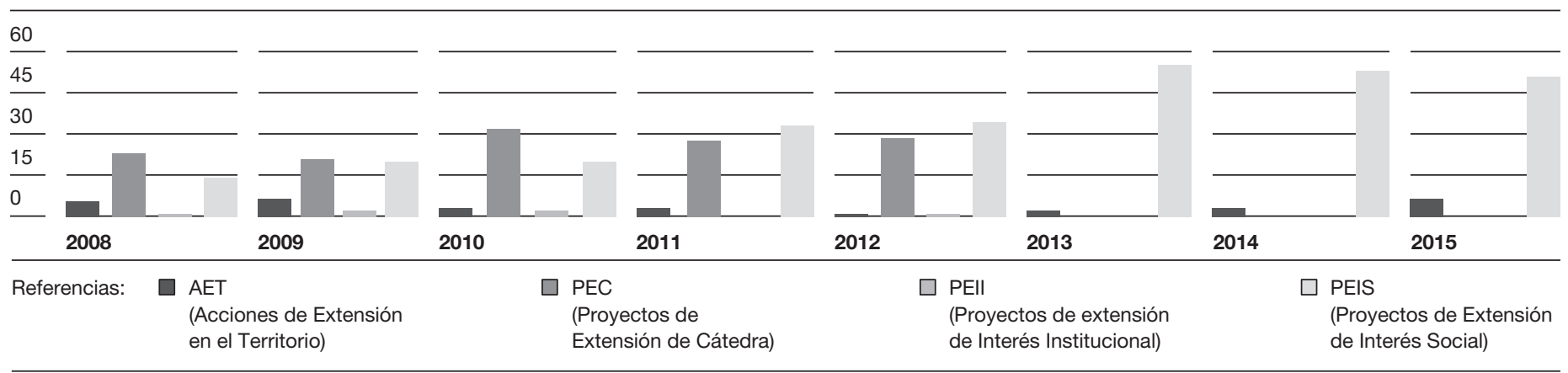

Fuente: Área de Planeamiento y Evaluación. Secretaría de Extensión, UNL. 
estar presentes para evaluar el proceso de un proyecto de extensión: dimensión sustancial (se refiere a los propósitos medulares del proyecto), gerencial o instrumental (las líneas de acción para lograr los propósitos medulares) y estratégica (las modalidades deseables de la gestión). Una vez definidas estas dimensiones, constituimos las variables de estructura, de procesos y de resultados, con las que evaluar los proyectos, tal como se sintetiza en el Cuadro 1: Dimensiones y variables.

Una vez que se rediseñó la herramienta para producir las evaluaciones, pudimos crear los indicadores. Así, los indicadores seleccionados para el análisis del accionar de los proyectos de extensión se agrupan en dos grandes tipologías: Intrínsecos y Relacionales. Dentro del primer grupo se encuentran: Coherencia interna (relación entre objetivos y resultados); Trabajo interdisciplinar; Comunicabilidad de los proyectos (en función de los materiales de comunicación utilizados en el transcurso de un proyecto). Dentro de este grupo se analizan las variaciones que cada uno presenta respecto de dos elementos distintivos: el programa de extensión en que se enmarca cada proyecto y su modalidad. De esta forma se observa la relación entre modalidad del proyecto y programa, y las diferencias (y continuidades) en los indicadores a partir de estos.

Por otra parte, dentro del tipo relacional se incluyen los siguientes puntos: relación entre participantes directos y el equipo de extensión; localización geográfica donde se desarrolla el proyecto; situación de los contextos; participación de las instituciones, (diferenciando entre participación activa o pasiva); ámbito de las instituciones intervinientes (públicas estatales nacionales, públicas estatales provinciales, públicas estatales municipales, públicas no estatales, privadas); tipo de instituciones intervinientes (instituciones educativas; municipios y comunas; ministerios y secretarías nacionales y provinciales; Organizaciones de la Sociedad Civil; vecinales; instituciones intermedias, sindicatos, colegios de profesionales, universidades, entre otras).

Cabe señalar que la relación entre variables y dimensiones no es estanca sino móvil, por lo que es posible ubicar una o más variables por dimensiones. Asimismo, que la construcción de indicadores se encuentra en proceso de revisión y, no obstante, se escogió compartir con los lectores los momentos por los que transita el Área de Planeamiento y Evaluación de la Secretaría de Extensión de la UNL.

El proceso evaluativo desarrollado se presenta como resultado de la revisión de lo actuado en determinado período y como insumo para diagramar y planificar los cursos de acción futura. La definición de los indicadores, y la consiguiente evaluación a partir de los resultados cuantificados, permiten no solo contrastar el accionar aislado de los proyectos de extensión sino, más importante aún, representan el principal insumo para la planificación de acciones futuras. Esta planificación tiene como sustento, entre otras cuestiones, la identificación de temáticas definidas como relevantes y no abordadas suficientemente hasta el momento; la delimitación de territorios considerados como prioritarios para el accionar extensionista; la detección de instituciones intervinientes cuyo potencial no ha sido capitalizado aún y la posibilidad de extenderse a otras intervenciones.

\section{Cuadro 1: Dimensiones y variables}

\begin{tabular}{|c|c|c|c|}
\hline Dimensiones & Variable de estructura & Variable de Procesos & Variables de Resultados \\
\hline $\begin{array}{l}\text { Sustancial (estructura del proyecto) } \\
\text { Hacen referencia a los propósitos } \\
\text { medulares de los programas o } \\
\text { proyectos. }\end{array}$ & Modalidad Programa & Temáticas & $\begin{array}{l}\text { Coherencia entre resultados y } \\
\text { objetivos. }\end{array}$ \\
\hline $\begin{array}{l}\text { Gerencial o instrumental (relación con } \\
\text { Instituciones y grado de participación) } \\
\text { Se vinculan a líneas de acción más } \\
\text { instrumentales y que constituyen los } \\
\text { medios para el desarrollo de las otras } \\
\text { líneas sustantivas. }\end{array}$ & Tipo de Institución & Interdisciplina & Tipo de participación \\
\hline $\begin{array}{l}\text { Estratégicas (integración entre actores } \\
\text { sociales directos al proyecto) } \\
\text { Se relacionan con las modalidades } \\
\text { deseables de la gestión social. }\end{array}$ & Temática & Material de difusión & $\begin{array}{l}\text { Tipo de relación entre equipo de } \\
\text { extensión y participantes directos }\end{array}$ \\
\hline
\end{tabular}

Fuente: elaboración propia. 
La construcción de indicadores para el análisis y evaluación de proyectos de extensión como procesos sociales es el desafío presente. De este modo, lograr consolidar un sistema de evaluación permanente permitiría fortalecer los lineamientos teórico-políticos de la extensión y afianzarla como una parte necesaria para contribuir a la apropiación social de los conocimientos y, por ende, a la idea de transformación y cambio.

\section{Reflexiones finales}

Se considera que las intervenciones de extensión que se realizan en los espacios sociales deben implicar necesariamente la producción de conocimientos y estos tienen que ser socialmente acordados. La cohesión de esta producción conlleva la potencia de transformación. Si en el proceso de conocimiento no se logra o se pretende lograr una transformación, no se podría estar hablando de la extensión como la producción de conocimientos socialmente acordados.

Desde un punto de vista filosófico, se propone pensar el proceso de producción/transformación de conocimientos como voluntad de potencia. Pensar la extensión universitaria de esta manera implica pensar, planificar acciones en las que se enmarque una voluntad de transformación material del espacio y por lo tanto de los agentes/actores involucrados en los procesos sociales. Ser parte y continuar dispuestos a la complejidad que implica la extensión universitaria es una apuesta filosófica de "voluntad de potencia". La voluntad, para Nietzsche, no está fuera del mundo, ella se da, se genera en relación. Esto significa que la voluntad es múltiple y se muestra como efectivamente real. Al mismo tiempo que la voluntad de potencia se enuncia siempre en plural. Y aquí radicaría el ser de la extensión universitaria, pues ella ES siendo plural. Si la extensión universitaria es un proceso de producción/ transformación del conocimiento, se puede inferir que es voluntad de potencia también, puesto que es la que busca expandirse, superarse, juntarse con otras potencias para tornarse mayor. La extensión universitaria, tal como aquí se plantea, implica un trabajo, una intervención, en un campo de inestabilidad y lucha en donde se entrama un juego constante de fuerzas. Cabe decir que la extensión debería ser un mundo donde reine la diferencia, donde la fuerza sea superación, sea ese constante ir más allá de los propios límites.

\section{Referencias bibliográficas}

Augé, M. (2000): Los no lugares. Espacios del anonimato. Una antropología de la sobre modernidad. Buenos Aires: Gedisa.

Bourdieu, P. (1984). El Espacio social y la génesis de las "clases". Recuperado de: http://www.culturascontemporaneas.com/contenidos/espacio_social_y_genesis. pdf (consultado en marzo de 2017).

Camilloni, A. de (2016). La evaluación de proyectos de extensión incluidos en el currículo universitario. Revista +E, (6), 24-35. Santa Fe, Argentina: Ediciones UNL. Carballeda, A. (2005). Las Cartografías Sociales y el territorio de la Intervención. Recuperado de: http://www.margen.org/carballeda/Las\%20Cartografias\%20Sociales.doc (consultado en marzo de 2017).

Cohen, E.; Franco, R. (2012 [1992]). Evaluación de Proyectos Sociales. Buenos Aires: Siglo XXI Editores.

Di Virgilio, M. y Solano, R. (2012). Monitoreo y evaluación de políticas, programas y proyectos sociales. Buenos Aires: Fundación CIPPEC.

Giménez, G. (1997). La Sociología de Pierre Boudeau. México: Instituto de Investigación Sociales de la Universidad Nacional Autónoma de México.

Matus, C. (1996). Planificar para gobernar: el método PES. Recuperado de: http:// cigob.org.ar/wp-content/uploads/2016/07/PES-para-web.pdf (consultado en marzo de 2017).

Menéndez, G. et al. (2013). Integración docencia y extensión. Otra forma de enseñar y de aprender. Santa Fe, Argentina: Ediciones UNL.

Nirenberg, O.; Ruiz, V.; y Brawerman, J. (2005). Evaluar para la transformación. Innovaciones en la evaluación de programas y proyectos sociales. Buenos Aires: Paidós. UNL (2010). "Línea de Orientación Principal II: Calidad”. Universidad Nacional del Litoral. Recuperado de https://www.unl.edu.ar/la-institucion/linea-de-orientacion-principal-ii-calidad/

- (2017a). Informes institucionales UNL. Recuperado de: http://www.unl.edu. ar/la-institucion/2017/03/10/plan-de-desarrollo-institucional-2010-2019/ (consultado en marzo de 2017).

(2017b). Informe de Autoevaluación. Recuperado de: http://www.unl.edu.ar/ blog/2017/07/03/tercera-evaluacion-institucional/ (consultado en marzo de 2017). Xinia Picado, G. (1997). Hacia la elaboración de indicadores de evaluación. Recuperado de: http://www.ts.ucr.ac.cr/binarios/docente/pd-000088.pdf (consultado en marzo de 2017). 


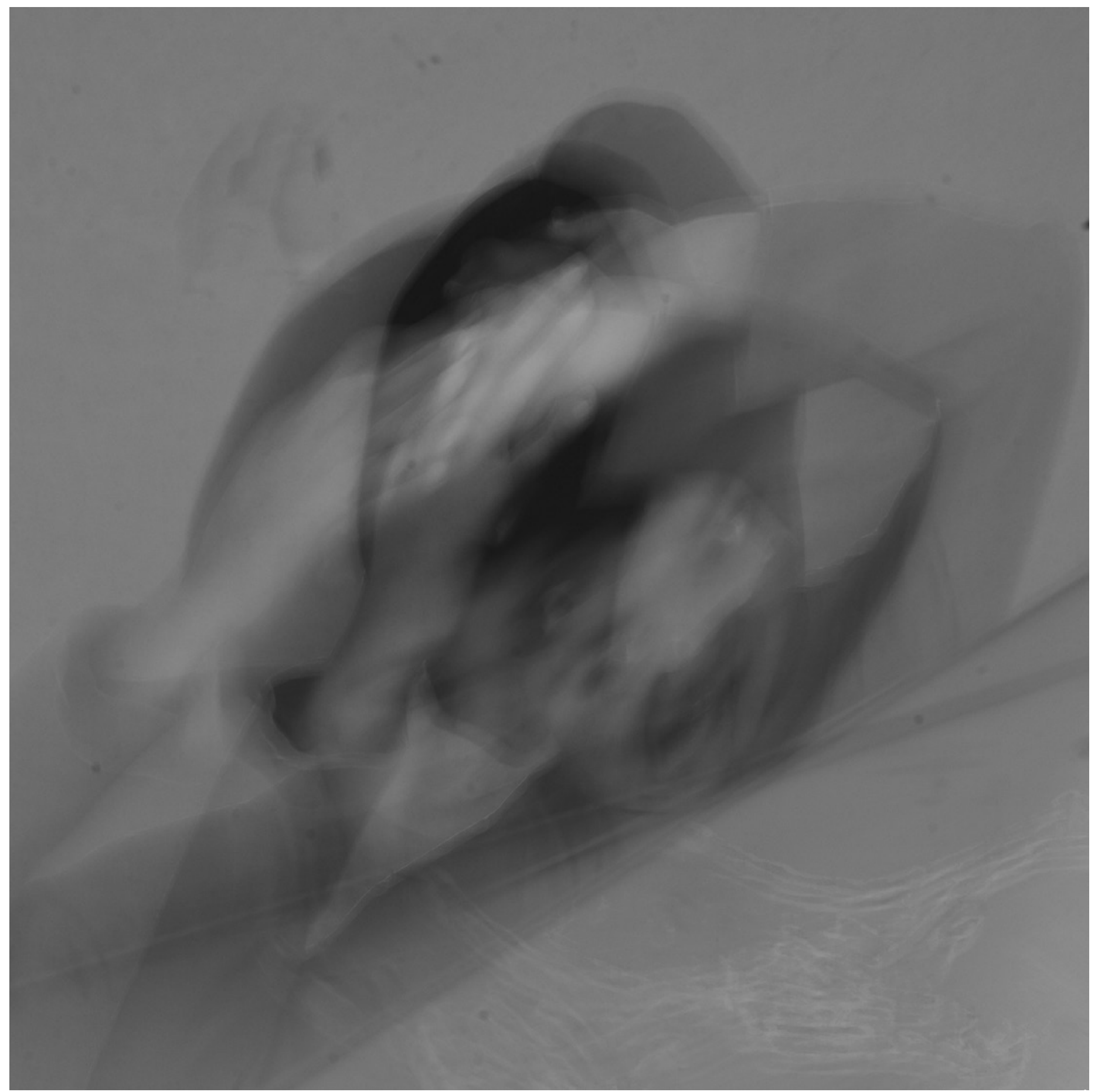

(c) Hugo Pascucci 